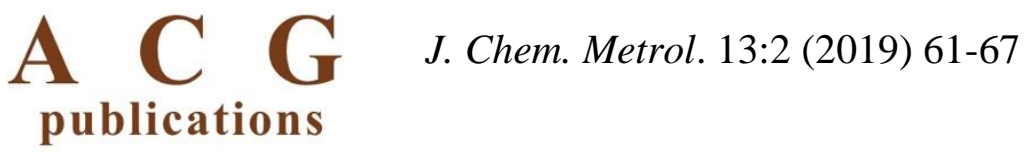

journal of chemical metrology

\title{
A GC-MS method for illegal stimulant drugs from serum : a multi-drug use sample in Turkey
}

\author{
Zeynep Turkmen $\oplus^{1 *}$, Merve Kuloglu $\odot 1$, Tugba Tekin $\odot 1$, \\ Selda Mercan ${ }^{1}$ and Isil Bavunoglu ${ }^{2}$ \\ ${ }^{1}$ Istanbul University-Cerrahpasa, Institute of Forensic Sciences and Legal Medicine, 34500, \\ Hadimkoy Campus, Büyükçekmece, Istanbul, Türkiye \\ ${ }^{2}$ Department of Internal Medicine, Cerrahpasa Medical Faculty, Istanbul University- \\ Cerrahpasa 34098, Cerrahpasa, Istanbul, Türkiye
}

(Received November 24,2019; Revised Deember 21,2019; Accepted December 24,2019)

\begin{abstract}
It is rather difficult to determine simultaneously more than one drug from different biological samples and to interpret the clinical picture in the case of multiple drug abuse. All mentioned drugs are life-threatening solely and the risk is becoming higher when they are used together. In this study, a gas chromatography-mass spectrometry (GC-MS) method was developed and validated for the simultaneous detection and quantification of amphetamine (AMP), 3,4-Methylenedioxymethamphetamine (MDMA) and cocaine in serum sample. The analytes were extracted by solid-phase extraction (SPE) cartridges, derivated with heptafluorobutyric anhydride at $60^{\circ} \mathrm{C}$ for $30 \mathrm{~min}$ and analyzed by GC-MS. Correlation coefficient values were found satisfactory. Linearity ranged from 1 to $20 \mu \mathrm{g} / \mathrm{mL}$ for all three substances. After acceptable results were obtained from calibration solutions, the study was conducted to observe matrix-match calibration and linearity parameters. Extraction efficiency of the method was observed in two concentrations spiked to serum matrix ( 5 and $10 \mu \mathrm{g} / \mathrm{mL}$ ) and found over $80 \%$ for three illicit drugs. Precision, accuracy, stability evaluation were appropriate as well. The validated analytical method was applied to an authentic sample which obtained from a patient administered to emergency room. The patient was suspected in regard to multi-drug use of stimulants and serum sample was analyzed by the presented method and AMP, MDMA and cocaine use was determined with concentrations of $0.45,1.03$ and $1.98 \mathrm{mg} / \mathrm{mL}$ in blood respectively. The developed method proved capable of quantifying simultaneous AMP, MDMA and cocaine use proposed in this study. Multidrug abuse is not a novel phenomenon but has to be determined as soon as possible by forensic toxicology laboratories because of potential severe health risks.
\end{abstract}

Keywords: Forensic toxicology; multi drug abuse; amphetamine; MDMA; cocaine; GC-MS. C2019 ACG Publication. All right reserved.

\section{Introduction}

Narcotic drugs are substances that have been abused for centuries and have addictive potential with suppressive or stimulant effects of the central nervous system. Heroin, marijuana, cocaine, and ecstasy are the most frequently used conventional illicit drugs, which MDMA and cocaine as stimulants have high addictive properties [1-3].

\footnotetext{
*Corresponding author E-Mail: zeytur@gmail.com
} 
Recently, studies on identifying drug-related trends have shown an increase in the simultaneous use of multi drugs especially in recreational club and school area among young people. Different intake of illicit drugs and derivatives are continuing to be the most important problem for public health [1]. In particular, more than half of drug abusers who have admitted to the Emergency Departmen (ED) due to acute toxicity are declared the multi-drug addiction behavior. Although opiates have a central position in this multi drug abuse, co-administration of MDMA and cocaine is still the most common problem in ED.

In the production of illicit substances and due to the hidden nature of the market, consumed drugs may contain a number of psychoactive substances whose interactions are less known and potentially dangerous. Since 2000 , over $45 \%$ of overdose deaths have been caused by multiple illegal drug use. Although this ratio varies to countries, it is increasing gradually. This is highlighted in the latest reports of the European Monitoring Center for Drugs and Drug Addiction (EMCDDA). Poor physical and mental health, bad living conditions, high risk of infection and the use of more than one substance have been reported to make this group susceptible to a range of chronic health problems such as cardiovascular, pulmonary and hepatic [4].

According to EMCDDA reports, there is no exact information about the frequency of multi drug abuser since abusers are categorized under "other substances" list [4]. The abuse of multi drug has a higher health risks than any one of these drugs abused separately [5]. In addition, the abuse of these drugs as cocktail form causes severe psychiatric disturbances and unsuccessful addiction treatment in comparison to single drug abuse [6-8].

It is rather difficult to simultaneously determine more than one drug from different biological samples and to interpret the clinical picture in the case of multiple drug abuse. Blood (including serum and plasma) is the most proper biological sample that reflects the exact drug concentration. Therefore, the most important biological evidence to reach more accurate data than the survey studies in determining the tendency in multi-drug abuse is blood, and analysis of the blood sample is necessary to get the answer to similar questions [9].

Simultaneous use of cocaine and ecstasy is common in the party or clubbing culture, especially among young adults [10]. All mentioned drugs are life-threatening solely and the risk is becoming higher when they are used together. Amongst the most dangerous side effects of cocaine use are as follows: Sudden death (can occur even during the first time), heart attack, cardiac arrhythmias, erratic behavior, paranoia, tremors, strokes, seizures, coma [7].

Ecstasy and cocaine are oftentimes used concurrently in club or party settings among young adults and can cause severe symptoms even death. Although cocaine is not the most commonly used drug in combination with ecstasy, $34 \%$ of one study's ecstasy-using participants reported having used cocaine together with ecstasy [10]. Furthermore, another study found that ecstasy users are far more likely to use cocaine than marijuana users; $46 \%$ of MDMA abusers used cocaine while only $2 \%$ of marijuana users abused cocaine [5].

According to the most recent statistics, ER visits from MDMA increased 123\% from 2005 to 2009. What makes MDMA particularly dangerous is that it the drug itself is highly variable; it is often contaminated with other substances including amphetamines, cocaine, caffeine, PMA and PCP, which increase the risk for adverse effects [11].

Studies have revealed that long-term use of amphetamine-type stimulants together with cocaine may cause changes in brain reward mechanism and behavior [12]. The brain regions that regulate the decision-making process and impulsivity also vary with the use of these substances. In the case of multidrug use habit continues, tendency to repeat the behavioral changes increase [12]. Cocaine and MDMA, as stimulants, are known to cause an increase in blood pressure, body temperature and heart rate leading to organ failure and death [12]. At present, there are no FDA approved drug-based, or medical therapies to treat cocaine/MDMA abuse. However, there are some promising developments in this area [13].

In this study, a GC-MS method was developed to determine the substances in the serum sample for the detection of amphetamine (AMP), 3,4-Methylenedioxymethamphetamine (MDMA) and cocaine for multidrug abusers. This developed method was applied to a patient who was suspected of substance abuse in the emergency department. The validity data of the method was evaluated with the patient sample. 


\section{Experimental}

\subsection{Chemicals and Instrumentation}

The cocaine, amphetamine and MDMA standards were supplied from LGC-Standards (London, UK). Methanol and dichloromethane were purchased from Merck (99,8-100\%, Darmstadt, Germany). All solvents used were of GC grade. Sodium hydroxide, ethyl acetate, n-heptane and hydrochloric acid (37\%) were purchased from Sigma Aldrich (St. Louis, Missouri, ABD). Oasis HLB (60 mg, $3 \mathrm{~mL}$ ) Solid phase extraction (SPE) cartridges were purchased from Waters (Milford, MA, USA). Heptafluorobutyric anhydride (HFBA) was also supplied from Sigma (St. Louis, Mo, USA)

Agilent HP 7820A Gas Chromatography device and 5977E Mass Spectrometer (USA), equipped with a $30 \mathrm{~m} \times 0.25 \mathrm{~mm}$ fused silica capillary column (HP-5MS 5\% Phenyl Methyl Siloxane, film thickness $0.25 \mu \mathrm{m}$ - Agilent Part No. 19091S-433) were used to perform analytical measurements. The experiments were conducted with Helium gas ( $>99.999 \%$ purity, Okser, Turkey). Evaporation under nitrogen stream was conducted with a HyperVap HV-300 from Gyrozen (Daejeon, Rep. of Korea) during sample preparation. A Direct-Q UV 3 ultrapure water system was acquired from Millipore (Molsheim, France) $(18.2 \mathrm{M} \Omega \mathrm{cm})$. SPE negative pressure vacuum manifolds with 24 ports were used for the extraction of the samples. The splitless mode was used with $1 \mu \mathrm{L}$ samples being injected. Total run time was $24.50 \mathrm{~min}$. Temperatures of MS analyzer and MS Ion Source were setup $150^{\circ} \mathrm{C}$ and $230^{\circ} \mathrm{C}$, respectively. The oven temperature was programmed from $55^{\circ} \mathrm{C}(2 \mathrm{~min}$ hold $)$ to $250^{\circ} \mathrm{C}$ at $10^{\circ} \mathrm{C} / \mathrm{min}(2$ min hold).

\subsection{Method Optimization and Sample Preparation}

In this study the selectivity/specificity, limit of detection (LOD), limit of quantification (LOQ), linearity, precision, accuracy, recovery and stability parameters were assayed. The calibrators and spiked samples were prepared by addition known amounts of analytes to the drug-free serum used as blank matrix.

Stock solutions of amphetamine, MDMA and cocaine reference standards were prepared at 1000 $\mu \mathrm{g} / \mathrm{mL}$ in $\mathrm{MeOH}$ and the stock solutions were used to obtain mixed working solutions containing all targeted substances in order to achieve the calibration curves. Working solution concentrations were 1 , $2,5,10$ and $20 \mu \mathrm{g} / \mathrm{mL}$ for all analytes. Each calibration points were analyzed six times and calibration curve was generated. To check out solvent response, blank solution consisting of only $\mathrm{MeOH}$ was also analyzed six times.

After linearity studies conducted with methanol solutions, same calibration ranges were also applied to serum samples to obtain matrix-matched calibration curve. Each calibration points were analyzed for six times including blank serum sample. All blood samples were collected from both the patient and non-abuser volunteer with the approval of ethical committee of Cerrahpaşa Medical Faculty and informed consents were obtained during sample collection (Approval No:10291). Precision, accuracy and recovery studies were assessed by evaluating six replicates of two calibration points (5 and $10 \mu \mathrm{g} / \mathrm{mL}$ ) which spiked to serum samples and standard deviation (SD), relative standard deviation (RSD\%) and bias\% were calculated to evaluate the method performance. LOD and LOQ values were determined according to signal-to-noise ratio of 3.3 and 10 respectively. To check out the stability, extracted samples kept at room temperature were analyzed after a week again and differences were evaluated.

In sample preparation step, the previously published method by Mercan et al. was modified to serum matrix [14]. Oasis-HLB SPE cartridges were used for extraction of analytes from $200 \mu \mathrm{L}$ serum samples. After extraction process eluates were gently evaporated to dryness at ambient temperature under nitrogen. For derivatization step, dried samples were reconstituted in $20 \mu \mathrm{L}$ of acetonitrile and transferred to glass inserts in autosampler vials. $50 \mu \mathrm{L}$ of derivatizing reagent HFBA and $50 \mu \mathrm{L}$ of ethylacetate was added and vials were kept at $60^{\circ} \mathrm{C}$ for $30 \mathrm{~min}$, and samples were dried with slow stream 
of nitrogen again. $200 \mu \mathrm{L}$ of ethylacetate was added to each insert for reconstitution and injection volume was set as $1 \mu \mathrm{L}$ for Gas Chromatography-Mass Spectrometry (GC-MS) analysis.

After optimization and validation studies, an authentic serum sample was experimented according to the above mentioned extraction procedure. Sample was obtained a patient who has administered to the emergency room with several symptoms such as tachycardia, sweating and excitation [15]. After detailed anamnesis, emergency clinician decided to investigate the case in terms of illicit drug abuse and sent the biological sample to the forensic toxicology laboratory.

\subsection{GC-MS Analysis}

The retention time of the compounds in spike serum samples was obtained as $13.35 \pm 0.03 \mathrm{~min}$ for AMP-heptaflouryl, 16.25 $\pm 0.04 \mathrm{~min}$ for MDMA-heptaflouryl, and 20.87 \pm 0.04 min for cocaine, as shown in the total ion chromatogram in Figure 1. Detected ions for analytes were as follows; 91, 118 and 240 for AMP-heptaflouryl; 162, 210 and 254 for MDMA-heptafloryl; 82, 182 and 303 for cocaine (Quantitation ions were underlined).

\section{Results and Discussion}

AMP, MDMA and cocaine were analyzed simultaneously by a validated GC-MS method in scanning mode. The three most abundant ions for each analyte were preselected from electron-impact spectra. Extracts were free of interferences at the appropriate retention times of each analyte. An entire run time was found long enough to condition the column and prevent carry over. Resolution of peaks and matrix effect were quite good both in methanol and serum matrices. Peak shapes were in good form and signal to noise ratios were acceptable as well.

After analysis of both calibration solutions and matrix-match calibration samples six times including blank sample, correlation coefficients of all quantitatively determined 3 analytes were satisfactorily found $\mathrm{R} \geq 0.996$ and calibration curves were obtained according to linear regression analysis. Linear ranges were obtained as $1-20 \mu \mathrm{g} / \mathrm{mL}$ for each three analytes in both linearity studies. LOQ levels generated from ten fold of signal to noise ratio were determined as $0.90,0.80$ and $0.75 \mu \mathrm{g} / \mathrm{mL}$ for AMP, MDMA and cocaine respectively. Recovery, precision and accuracy results obtained from two different spiked concentrations were found satisfied and stability of each analytes were found acceptable as well. Recoveries of cocaine were $>92 \%$ for $5 \mu \mathrm{g} / \mathrm{mL}$ and $96.9 \%$ for $10 \mu \mathrm{g} / \mathrm{mL}$, whereas recovery of MDMA and AMP as less polar compounds were found between 80.6-85.5\% for two concentration levels. Within-run and between-run, relative accuracies for cocaine were $>90 \%$ with 9.22 mean CV\%, but AMP and MDMA's within-run determinations were ranged from 11.9-12.4 CV\%. Stability of analytes, detailed method performance results including RSD\%, recovery $\%, \mathrm{CV} \%$, correlation coefficients of calibration curves etc. were presented in Table 1.

According to validation results, it is revealed that three stimulant illegal drugs can be easily detected from serum sample with this derivated solid phase extraction and GC-MS method. Considering recoveries, over $82.2 \%$ found in the presented method is acceptable extraction efficiency for related compounds. In a study conducted by Pelição, F. S. et al., lower values (74\%) were obtained for the same analytes. However LOQ levels were found higher in our presented study when compared similar studies [16]. By increasing extraction volume and concentrate the analyte before analysis, it is possible to detect low amount of drugs as well, since the matrix effect was not observed in blank serum sample. Also consistency of analytes was found successful according to the standard deviation of retention times. Linearity of all three analtyes was found over 0.996 correlation coefficient and better which are acceptable for analytical studies. Although the derivatization of AMP and MDMA is an additional step during extraction procedure, this process enhanced both the sensitivity and LOQ. At the same time HFBA is inert for cocaine and does not affect or interact the results of cocaine. 
Table 1. Results of method performance parameters

\begin{tabular}{lccc}
\hline Parameters & AMP-HFBA & MDMA-HFBA & Cocaine \\
\hline Retention time $(\mathrm{min} ; \pm \mathrm{SD})$ & $13.35 \pm 0.03$ & $16.25 \pm 0.04$ & $20.87 \pm 0.04$ \\
Linear range $(\mu \mathrm{g} / \mathrm{mL})$ & $1-20$ & $1-20$ & $1-20$ \\
Correlation coefficient $\left(\mathrm{r}^{2}\right)$ & 0.996 & 0.996 & 0.997 \\
LOQ $(\mu \mathrm{g} / \mathrm{mL})$ & 0.90 & 0.80 & 0.75 \\
Recovery $(\%)$ & 80.6 & 84.4 & 92.8 \\
$(5 \mu \mathrm{g} / \mathrm{mL}, \mathrm{n}=6)$ & & & \\
Recovery $(\%)$ & 82.2 & 85.5 & 96.9 \\
$(10 \mu \mathrm{g} / \mathrm{mL}, \mathrm{n}=6)$ & & & \\
RSD\% (mean) $(\mathrm{n}=12)$ & 6.78 & 5.09 & 4.49 \\
CV\% (mean) $(\mathrm{n}=12)$ & 12.4 & 11.9 & 9.22
\end{tabular}

SD: standard deviation; RSD: relative standard deviation; $\mathrm{CV}$ : correlation variation

Considering the patient's serum sample, AMP was found $0.45 \mathrm{mg} / \mathrm{mL}$, MDMA was found 1.03 and cocaine was found $1.98 \mathrm{mg} / \mathrm{mL}$ in final concentration according to the quantitation and back calculation of the total ion chromatogram as shown in Figure 1.

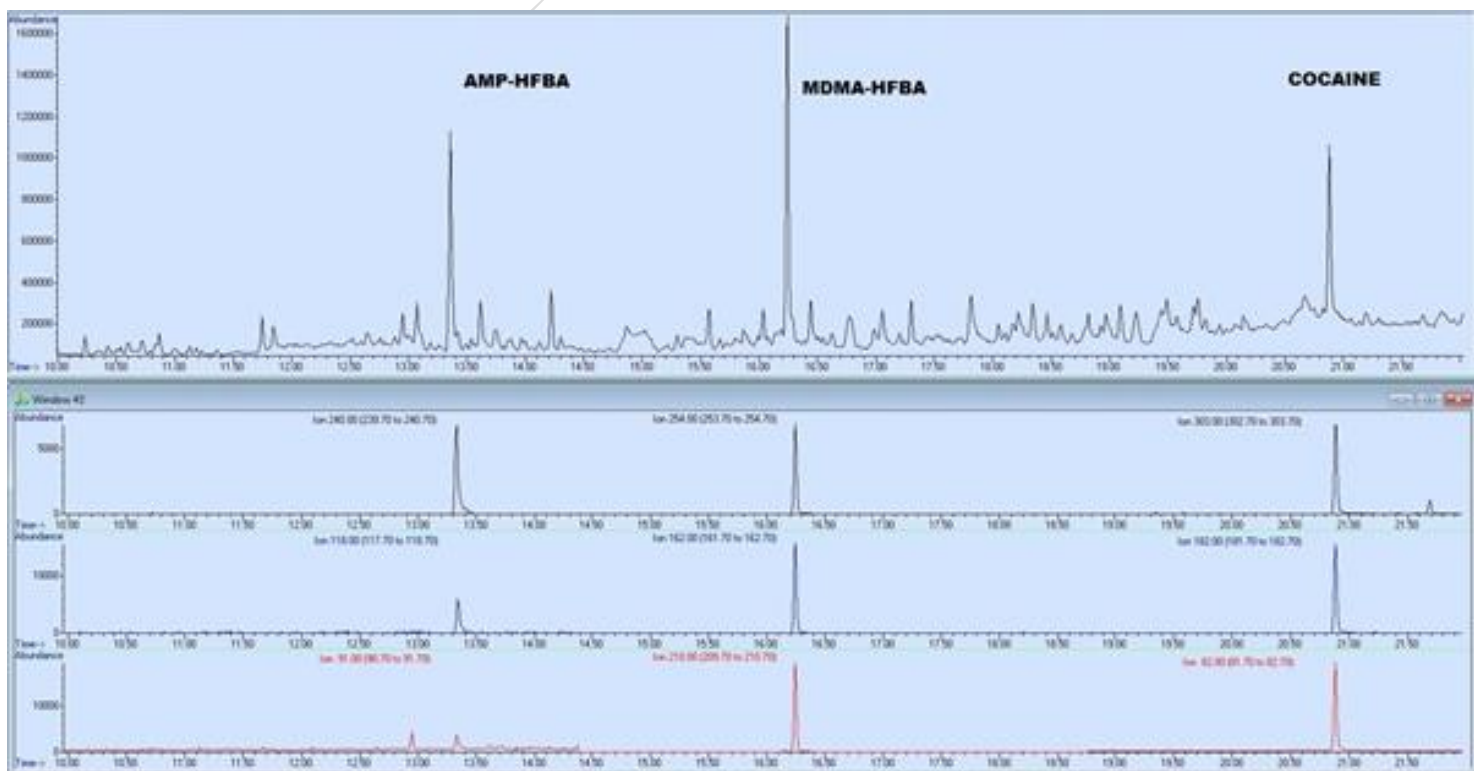

Figure 1. The chromatogram of patient's serum sample and mass spectra of presenting multidrug abuse including amphetamine, MDMA and cocaine simultaneously

There are several studies conducted with alternative matrices such as hair and urine for determination of amphetamine-type stimulant via HFBA derivatization in the literature. In a related study, amphetamine, methamphetamine, methylenedioxyamphetamine, MDMA and norketamine in hair were determined by GCMS, with HFBA derivatization at $50{ }^{\circ} \mathrm{C}$ for $30 \mathrm{~min}$ and in this method DB$5 \mathrm{MS}$ column is used with a $10 \mathrm{~min}$ run time by similar GC-MS instrument condition. It is stated that the developed method had a linear range of $0.1-10 \mathrm{ng} / \mathrm{mL}$ for each compounds and the LOD values were determined higher than $0.028 \mathrm{ng} / \mathrm{mg}$ in hair [17]. In another similar study, the GC-MS method has been developed for the same compounds besides ketamine in urine sample with HFBA derivatization at 60$75{ }^{\circ} \mathrm{C}$ for $30 \mathrm{~min}$ and similar characteristics were obtained with GC-MS instrument with 10 min runtime on HP-1MS column. The maximum level of the concentrations of related compounds with the developed method were found $8 \mu \mathrm{g} / \mathrm{mL}$ for amphetamine type drugs and $6 \mu \mathrm{g} / \mathrm{mL}$ ketamine and its derivatives. Also the stability of derivatized amphetamine compounds has been investigated under different temperature $\left(60-100{ }^{\circ} \mathrm{C}\right)$ with HFBA acylation [18]. According to results and evaluation of related researches, HFBA derivatization is supposed as the best way to enhance the efficiency of 
amphetamine type drugs as also revealed in this presented study. Additionally, LOQ levels and other performance criteria of the method shown similarity to the literature [17-19].

It has also seen that fluorescence derivative 4- (4,5-diphenyl-1H-imidazole-2-yl) benzoyl chloride (DIB-Cl) was also used for the detection of ecstasy group compounds from biological matrices besides GC derivatization agents. It has been reported in a different study that the phenyl alkyl amines containing samples with low concentration levels, such as hair were extracted, derivatized and analyzed by HPLC-FL for the identification of ecstasy users [20].

Ecstasy group has been the most popular group since its discovered end of 1980. It is stated that 0.5-2 million tablets of ecstasy has been used in the UK annually. In particular, it is reported that $4.5 \%$ of people aged 15-34 have been reported to have used MDMA in the last 12 months. It is stated that a group of illicit substances with such intensive use, especially in anesthesia, intensive care and emergency departments, suffer from serious poisoning, organ losses and may even result in death [15].

In spite of the intense consumption of MDMA, severe acute illness is relatively rare. Complications of MDMA intake with concomitant use of other drugs or alcohol may be life-threatening and in this case it may be necessary to approach the patient with a well-planned treatment for the physiological effects and toxicity profile of the substance based on its clinical condition and anamnesis. It should be noted that there are still many unanswered questions about the pathophysiology and pharmacology of acute toxic effects of MDMA. It is clear that many different neuroendocrine systems may be affected and the variety of side effects may be due to many factors, such as environmental and pharmacogenetic differences. In addition, the possibility of permanent damage to serotonergic neurological pathways in MDMA users should never be forgotten [15]. As for cocaine, its usage can commonly cause health problems especially cardiovascular, cerebrovascular and neurological problems [4].

\section{Conclusions}

In this work, a simple and selective analytical method was successfully developed and validated simultaneously for the detection and quantitation of AMP, MDMA and cocaine in serum sample and applied to an authentic sample delivered to forensic toxicology laboratory from a multidrug abuser in an emergency room. The method was demonstrated to be highly reproducible, specific, precise, and accurate and the presented study suggested that validated method is proper for the simultaneous quantitation of AMP, MDMA, and cocaine in serum using HFBA derivatization with GC-MS using SPE. As stimulants all three substances are commonly abused in recreational manner especially by young adults. Short and long term effects of multidrug abuse is still under review and should be taken into consideration by relevant organizations governmental bodies.

\section{Acknowledgement}

This study was supported by Istanbul University-Cerrahpasa, Scientific Research Foundation with a project numbered YADOP-50936. The authors also would like to thank to Lab. Tech. Murat Yayla for his technical support.

\section{ORCID}

Zeynep Turkmen: $\underline{0000-0002-8250-7520}$

Merve Kuloglu: 0000-0002-3670-3168

Tugba Tekin: 0000-0001-6573-8426

Selda Mercan: 0000-0002-0431-6972

Isil Bavunoglu: 0000-0001-5146-2254

\section{References}

[1] S. Mercan and M. Acikkol (2014). Drug facilitated crimes: Drugs and effects; evidences and analysis: Review, Turkiye Klinik. J. Foren. Sci. Leg. Med. 11(2), 78-96. 
[2] M. Acikkol, S. Mercan and N. Ziyalar (2011). A Forensic and social approach to drug-facilitated crimes, crime rates, types and hot spot, ed: Alexander D. Morina, Nova Science Publisher, United Kingdom, Chapter 1, pp.1-42.

[3] W.M. Mohamed, S.B. Hamida, J.C. Cassel, A.P. De Vasconcelos and B.C. Jones (2011). MDMA: Interactions with other psychoactive drugs, Pharmacol. Biochem. Behav. 99(4), 759-774.

[4] http://www.emcdda.europa.eu/system/files/publications/982/Drugnet_Europe90_web_optimised_Final.p $\underline{\mathrm{df}}$ (last access 08.12.2019)

[5] M.W. Boeri, C.E. Sterk, M. Bahora and K.W. Elifson (2008). Polydrug use among ecstasy users: separate, synergistic, and indiscriminate patterns, J. Drug Issues. 38 (2), 517 -541.

[6] J.J. Lipmann (1997) Recent forensic pharmacological developments in drug abuse: The growth and problems of speedballing, Forensic Exam. 6, 9-13.

[7] B.F. Sands and D.A. Ciraulo (1992). Cocaine drug-drug interactions, J. Clin. Psychopharmacol. 12, 4955 .

[8] S. Winbery, K. Blaho, B. Logan and S. Geraci (1998). Multiple cocaine-induced seizures and corresponding cocaine and metabolite concentrations, Am. J. Emerg. Med. 16, 529-533.

[9] H. Gjerde, E.L. Øiestad and A.S. Christophersen (2011). Using biological samples in epidemiological research on drugs of abuse, Norsk Epidemiologi. 21(1), 5-14.

[10] B.C. Kelly and J.T Parsons (2008). Predictors and comparisons of polydrug and non-polydrug cocaine use in club subcultures, Am. J. Drug Alcohol Abuse 34(6), 774-81.

[11] A.I. Qureshi, S.A. Chaudhry and M.F. Suri (2014). Cocaine use and the likelihood of cardiovascular and all- cause mortality: data from the third national health and nutrition examination survey mortality followup study, J. Vasc. Interv. Neurol. 7(1), 76-82.

[12] H. Uosukainen, U. Tacke and A.R. Winstock (2015). Self-reported prevalence of dependence of MDMA compared to cocaine, mephedrone and ketamine among a sample of recreational poly-drug users, Int. J. Drug Policy. 26(1), 78-83.

[13] C.M. Hysek, L.D. Simmler, V.G. Nicola, N. Vischer, M. Donzelli, S. Krähenbühl and M.E. Liechti (2012). Duloxetine inhibits effects of MDMA ("ecstasy") in vitro and in humans in a randomized placebo-controlled laboratory study, PloS One. 7(5), e36476. doi:10.1371/journal.pone.0036476.

[14] S. Mercan, M. Kuloglu T. Tekin, Z. Turkmen, A.O. Dogru, A.N. Safran and F. Asicioglu (2019). Wastewater-based monitoring of illicit drug consumption in Istanbul: Preliminary results from two districts, Sci. Total Environ. 656, 231-238.

[15] A.P. Hall and J.A. Henry (2006). Acute toxic effects of 'Ecstasy' (MDMA) and related compounds: overview of pathophysiology and clinical management, Br. J. Anaesth. 96(6), 678-685.

[16] F.S. Pelição, M.D. Peres, J.F. Pissinate and B.S. De Martinis (2014). A one-step extraction procedure for the screening of cocaine, amphetamines and cannabinoids in postmortem blood samples, J. Anal. Toxicol, 38(6), 341-348.

[17] J.Y. Kim, S.H. Shin, J.I. Lee and M.K In (2010). Rapid and simple determination of psychotropic phenylalkylamine derivatives in human hair by gas chromatography-mass spectrometry using micro-pulverized extraction, For. Sci. Int. 196(1-3), 43-50.

[18] H.H. Lee, J.F. Lee, S.Y. Lin, P.H. Chen and B.H. Chen (2011). Simultaneous determination of HFBAderivatized amphetamines and ketamines in urine by gas chromatography-mass spectrometry, J. Anal. Toxicol. 35(3), 162-169.

[19] J. Segura, R. Ventura and C. Jurado (1998) Derivatization procedures for gas chromatographic-mass spectrometric determination of xenobiotics in biological samples, with special attention to drugs of abuse and doping agents, J. Chromatogr. B. 713, 61-90

[20] K. Nakashima, A. Kaddoumi, Y. Ishida, T. Itoh and K. Taki (2003). Determination of methamphetamine and amphetamine in abusers' plasma and hair samples with HPLC-FL, Biomed. Chromatogr. 17(7), 471476.

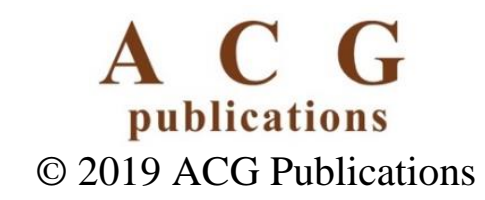

